\title{
Litiasis urinaria en adultos con mielomeningocele
}

\author{
Verdú Tartajo F, Salinas Casado J*, Herranz Amo F, Díez Cordero JM. Durán Merino R, \\ Hernández Fernández C. \\ Servicio de Urología. Hospital Universitario Gregorio Marañón. Madrid. \\ *Unidad de Urodinámica. Servicio de Urología. Hospital Clinico Universitario San Carlos. Madrid.
}

Actas Urol Esp. 2006;30(7):675-683

\section{RESUMEN}

LITIASIS URINARIA EN ADULTOS CON MIELOMENINGOCELE

Introducción y objetivos: Los avances en el tratamiento de los nacidos con mielomeningocele (MMC) han logrado un gran aumento en su supervivencia, permitiéndoles una longevidad nunca antes alcanzada, pero todavia son escasos los datos concernientes a los problemas urológicos que estos enfermos plantean durante su vida adulta. Hemos evaluado las características de la litiasis urinaria en adultos nacidos con MMC así como los tratamientos empleados en la misma.

Material y métodos: Revisamos 52 pacientes nacidos con MMC de entre 18 y 40 años, atendidos durante los últimos 14 años en nuestro hospital, con una media de seguimiento de 6,7 años.

Resultados: Se diagnosticaron de litiasis urinaria 10 pacientes $(19,2 \%)$. Tres formaron cálculos renales $(5,7 \%)$, y uno de ellos, junto con 7 pacientes más, desarrollaron cálculos vesicales (15,3\%). El nivel neurológico fue $\leq \mathrm{L}_{2}$ en 3 casos, $\mathrm{L}_{2}{ }^{-}$ $\mathrm{S}_{1}$ en $5, \mathrm{y} \geq \mathrm{S}_{1}$ en otros dos. El tipo de disfunción neurógena del tracto urinario inferior fue de lesión múltiple pura de neurona motora inferior en 6 casos, de neurona motora superior en 1, lesión múltiple mixta en otro, no pudiéndose valorar en el restante. Dos pacientes tenían una ampliación vesical y uno de estos junto con otros 3 pacientes era portador de AMS-800 no funcionantes. La litiasis vesical se trató endoscopicamente en 14 ocasiones y mediante cistolitotomía suprapúbica en 4, junto a la retirada de AMS-800 en 3 de ellas. Un paciente expulsó un pequeño cálculo. En un paciente fueron necesarias 2 litotricias extracorpóreas y 2 nefrolitotomías percutáneas. Tres pacientes tuvieron múltiples recidivas.

Conclusiones: La litiasis urinaria es frecuente en los adultos con MMC. Determinadas características de estos pacientes, junto con su configuración anatómica y algunos tratamientos empleados en ellos, ocasionan problemas de diagnostico, tratamiento y prevención de los cálculos que forman.

Palabras clave: Mielomeningocele. Adultos. Litiasis urinaria. Cálculos renales. Calculos vesicales

\section{ABSTRACT}

URINARY CALCULI IN MYELOMENINGOCELE ADULTS

Introduction and objectives: the improvements in the management of newborns with myelomeningocele (MMC) have obtained a big increase in survival, allowing them to get longevity like never before, but data regarding urologic diseases during adult age are still missing. We herein evaluate the features of urinary lithiasis in adults born with MMC and the therapies used for its treatment.

Material and method: we review 52 patients diagnosed at birth of MMC, between 18 and 40 years old, treated in our institution, with a mean follow-up of 6.7 years.

Results: 10 patients (19.2\%) were diagnosed of urolithiasis. Three developed kidney calculi and one of them, with 7 more patients, developed bladder calculi (15.3\%). The neurological level was $\leq$ L2 in 3 cases, L2-S1 in 5, and $\geq \mathrm{S} 1$ in the other two. The type of neurogenic dysfunction of inferior urinary tract was multiple lesion of lower motor neurone in 6 cases, upper motor neurone in 1 case, multiple mixed lesion in 1 case and in case it was impossible to determine. Two patients had a bladder augmentation procedure and one of these with other 3 patients had a non-functional AMS- 800 artificial urinary sphincter. Bladder stones were treated endoscopically in 14 procedures and by suprapubic cystolithotomy in 4 procedures, combined with removal of AMS-800 in 3 of them. One patient spontaneously passed a small stone. In one case, 2 ESWL and 2 percutaneous nephrolithotomies were needed. Three patients developed multiple recurrences during follow-up.

Conclusions: urinary lithiasis is common in adults with MMC. Some distinct features of these patients, together with their anatomical configuration and some therapies used in them, cause diagnostic, therapeutic and prophylaxis issues for the calculi they may develop.

Keywords: Myelomeningocele. Adults. Urolithiasis. Renal stones. Bladder calculi 
$\mathrm{E}^{1}$ mielomeningocele (MMC) es una malformación congénita del tubo neural, caracterizada por la falta de fusión de los arcos vertebrales posteriores, asociada a la extrusión de la médula y raíces nerviosas. Aunque el defecto puede aparecer a cualquier nivel de la columna, muestra predilección por el segmento lumbosacro y generalmente se asocia a hidrocefalia secundaria a una malformación de Arnold-Chiari. Su presencia, además de ocasionar alteraciones en los aparatos locomotor y digestivo, casi siempre ocasiona una alteración en la función del tracto urinario inferior.

La afectación neurógena del MMC varía ampliamente, dependiendo de los elementos contenidos en su saco meníngeo, por lo que puede causar distintos tipos de disfunción vesicouretral. Estos no guardan relación con el nivel óseo del defecto y precisan de estudios urodinámicos para su correcta filiación.

Teóricamente, pueden formarse cálculos en el aparato urinario de los pacientes nacidos con MMC, por la existencia de varios factores de riesgo asociados con su enfermedad. Así la presencia generalizada en los mismos de una disfunción vesical neurógena a menudo se acompaña de estasis e infección urinaria. Además la utilización de sondas y la interposición quirúrgica de segmentos intestinales en su tratamiento, favorecen la introducción de cuerpos extraños y la infección.

A lo largo de las últimas décadas, los avances médicos en el tratamiento de los pacientes con MMC, han logrado un gran aumento en su supervivencia y les han proporcionado una longevidad nunca antes alcanzada, permitiéndoles llegar a ser adultos. Pero sus necesidades asistenciales continúan y en gran parte se derivarán de sus problemas urológicos. El objetivo de este trabajo es contribuir al conocimiento de la litiasis urinaria en adultos con MMC, ya que estos pacientes, que presentan peculiaridades propias, serán cada vez más frecuentes en las consultas de urología.

\section{MATERIAL Y MÉTODOS}

Hemos revisado las historias clínicas de 52 pacientes nacidos con MMC y que han alcanzado la edad adulta, seguidos en nuestra unidad de urodinámica desde hace 14 años. El seguimiento medio de los pacientes fue de 6,7 años (intervalo de 1 a 14 años) y la edad media de los mismos al inicio de su control de 20.7 años (intervalo de 18 a 40 años).

El defecto óseo de la malformación, en la mayoría de los casos interesaba a las últimas vértebras lumbares y a casi la totalidad de las sacras. Las excepciones fueron 2 casos, con defectos limitados a su columna lumbar, y otro en el que el defecto lumbosacro se extendía a las vértebras dorsales. En todos los pacientes se determinó el nivel neurológico de su lesión, entendiendo como tal el segmento más caudal de la médula espinal con función normal en ambos hemicuerpos, y éste lo establecimos siguiendo el esquema que Schurtleff ${ }^{1}$ propuso, basándose en el nivel funcional motor, para el estudio de la problemática derivada de la supervivencia a largo plazo y de la integración social de los pacientes con mielomeningocele, pero sobre la base de los resultados de la exploración de la sensibilidad, dada la perdurabilidad del mismo, a diferencia del nivel motor, influenciable con el desuso, las contracturas y posibles cirugias, disponiéndose tres niveles de lesión: Nivel I, lesión en o por encima de $L_{2}$; Nivel II, lesión entre $L_{3}-L_{5}$; Nivel III, lesión en o por debajo de $\mathrm{S}_{1}$.

En todos los pacientes se realizaron estudios de imagen del aparato urinario generalmente consistentes en una urografia intravenosa al inicio de su seguimiento, seguido de un estudio radiológico simple del abdomen y ecografía urológica, con periodicidad según cada caso. En los pacientes en los que se consideró necesario se repitió el estudio urográfico o se complementó con otras técnicas de imagen.

A todos los pacientes se les realizó exploración neurourológica, determinando su sensibilidad perineal, tono y control voluntario anal, y reflejo bulbocavernoso junto con un estudio videourodinámico inicial, que se repitió periódicamente según el tipo de disfunción vesicouretral encontrada y la evolución clínica del paciente

Conforme a los resultados obtenidos de la exploración neurourológica y de los estudios videourodinámicos, diagnosticamos la lesión de los distintos componentes de la inervación vesicouretral para clasificar la disfunción neurógena del tracto urinario inferior de nuestros pacientes, en base al nivel de la lesión y al tipo de inervación lesionada ${ }^{2}$. 


\section{RESULTADOS}

En total 10 pacientes $(19,2 \%)$ se diagnosticaron de litiasis urinaria, durante su seguimiento desde el inicio de su vida adulta, de los cuales 4 son mujeres y 6 varones. Un paciente presentó cálculos renales, vesicales y prostáticos, 7 formaron cálculos vesicales y otros 2 cálculos renales (Tabla 1). La edad de los pacientes en el momento del diagnostico de litiasis osciló entre los 18 y 40 años, con una media de 23,7.

De los 3 pacientes con litiasis renal $(5,7 \%)$, uno tenía un cálculo piélico derecho y 2 tenían cálculos bilaterales, presentando además uno de estos litiasis vesical recidivante y múltiples cálculos prostáticos (Fig. 1). En todos ello se demostraba en sus estudios de imagen, una moderada ureteropielocaliectasia bilateral junto a un cistograma festoneado; en un caso existía reflujo vesicorrenal izquierdo.

El nivel neurológico de la lesión estaba en los tres, en o por encima de $\mathrm{L}_{2}$. Dos pacientes tenían arreflexia con mala acomodación vesical, con un sistema esfinteriano incompetente durante el llenado que permanecía fijo durante los intentos de vaciado. El tercer paciente presentaba hiperreflexia vesical y disinergia vesicoesfinteriana. La disfunción neurógena de la vía urinaria inferior de estos pacientes se catalogó como lesión múltiple pura de neurona motora inferior en dos de ellos y de neurona motora superior en el restante.

De la paciente con litiasis bilateral y reflujo se perdió el seguimiento, y el paciente con litiasis piélica falleció por intoxicación alimentaria antes de recibir tratamiento para su litiasis. En el tercer paciente realizamos dos sesiones de LEOC sobre un cálculo piélico izquierdo hasta comprobar la completa eliminación del mismo. Este mismo procedimiento fue imposible como tratamiento de su cálculo píelico derecho, dada la alteración en la posición de su riñón secundaria a la gran cifoescoliosis que presentaba (Fig. 2),

\section{Tabla 1}

Localización de los cálculos en 10 pacientes con mielomeningocele y litiasis urinaria, y porcentaje de cada tipo de litiasis, en 52 adultos con mielomeningocele

Pacientes con cálculos renales

Pacientes con cálculos renales y vesicales

Pacientes con cálculos vesicales

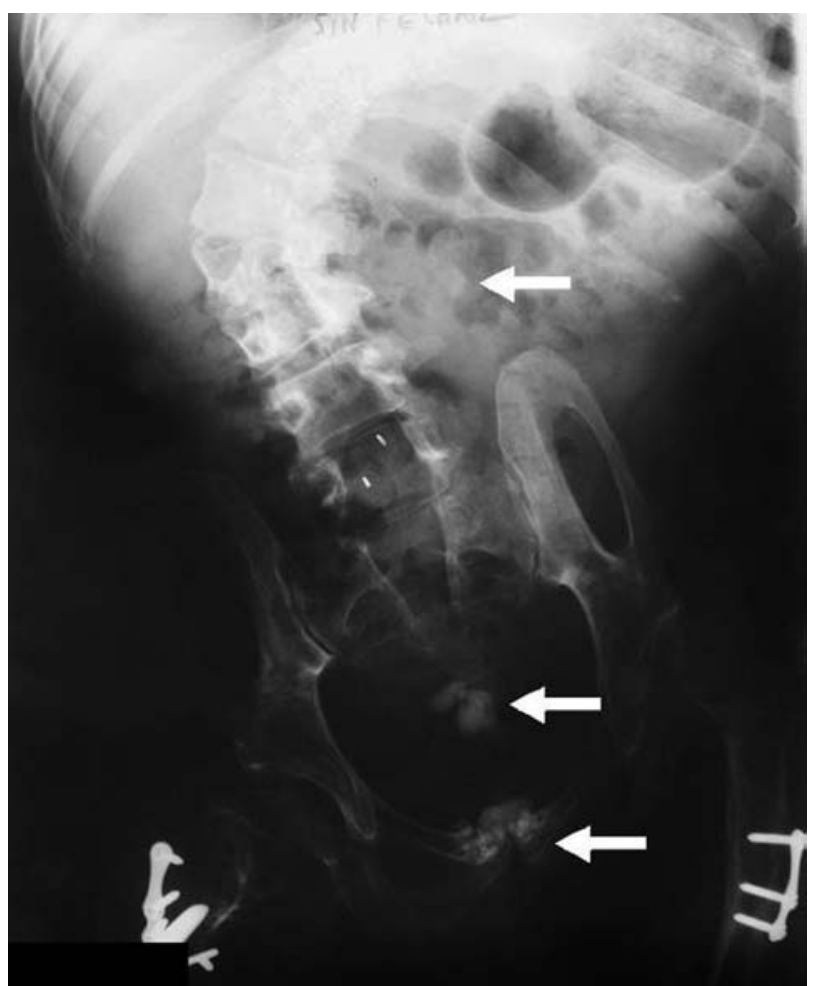

FIGURA 1. UIV. Litiasis renal, vesical y prostática. También se aprecian anormalidades óseas propias del MMC y material quirúrgico de corrección ortopédica.

por lo que se procedió a nefrolitotomía percutánea derecha, logrando la completa eliminación del cálculo. Un año después presentó recidiva de su litiasis píelica derecha, por lo que de nuevo repetimos el mismo procedimiento, logrando eliminar por completo el cálculo, cuyo análisis demostró una mezcla de fosfato cálcico y fosfato amónico magnésico.

De los 8 pacientes con litiasis vesical (15,3\%), sólo 1 (el paciente con litiasis renal y prostática) refería antecedentes litíasicos al habérsele realizado una cistolitotomía abierta a los 11 años. En ellos el diagnóstico de litiasis vesical se hizo en 19 ocasiones, puesto que en uno, que formó un cálculo a los dos años de su ampliación vesical, hizo una recidiva múltiple al año siguiente, en otro caso la litiasis recidivó en tres ocasiones y en un tercero existieron hasta 7 recidivas de sus cálculos vesicales favorecidas por la presencia de multitud de pequeñas litiasis engastadas en su parénquima prostático (Fig. 3). 


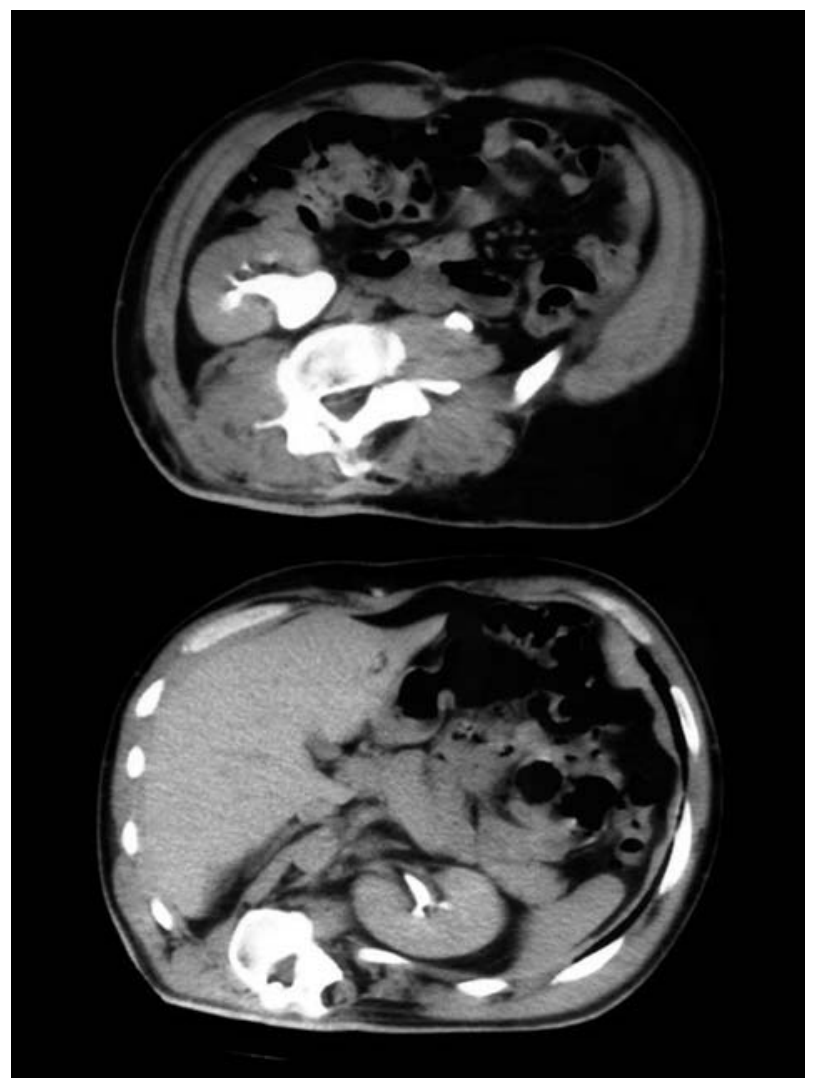

FIGURA 2. TAC. Localización anómala de ambos riñones, ocasionada por la presencia de una severa cifoescoliosis en un paciente con MMC.

En 4 casos los cálculos se formaron sobre componentes de un esfinter artificial (AMS-800) que no funcionaba, completa o parcialmente retirado, implantado durante su infancia. Uno de ellos se había colocado simultáneamente a la realización de una ampliación vesical con sigma detubulizado hacía siete años (Figs. 4 y 5). Otro cálculo se formó sobre el manguito de una prótesis emigrado a vejiga (Fig. 6) y un tercero sobre un manguito residual emigrado a vejiga. El último se formó sobre un pequeño resto de tubo de conexión de una prótesis de incontinencia erosionada a vejiga, que se retiró a la vez que se amplió la misma con sigma detubulizado, y que acabó emigrando a la neovegiga (Fig. 7).

Desde el punto de vista neurourológico, un paciente $(12,5 \%)$ tenía un nivel de lesión en o por encima de $\mathrm{L}_{2}$, cinco $(62,5 \%)$ entre $\mathrm{L}_{2}-\mathrm{S}_{1}$, y dos $(25 \%)$ en o por debajo de $S_{1}$. Cinco pacientes presentaban arreflexia vesical, con incompetencia esfinteriana durante el llenado y ausencia de relajación durante el vaciado. Una paciente tenía

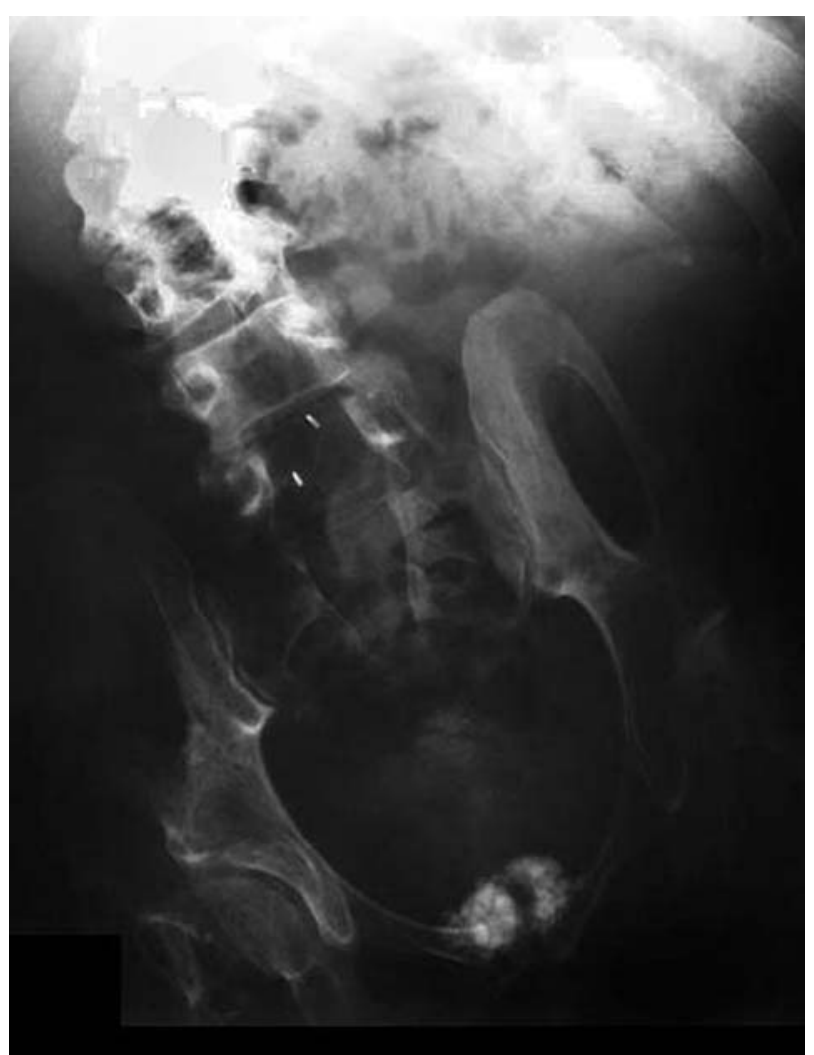

FIGURA 3. Placa simple de abdomen. Opacidad prostática secundaria a la presencia de multitud de pequeños cálculos intraparenquimatosos.

una vejiga arrefléxica con un sistema esfinteriano competente por la presencia de una prótesis AMS800 afuncionante, y los dos restantes tenían una vejiga de baja presión por ampliación vesical con sistemas esfinterianos competentes, en un caso por la presencia de una prótesis afuncionante y en el otro por fibrosis periuretral tras la retirada de un esfinter artificial. La disfunción neurógena de la vía urinaria de los pacientes con litiasis vesical correspondió a una lesión múltiple pura de neurona motora inferior en 6 casos (75\%); en otro caso $(12,5 \%)$ existía una lesión múltiple mixta (por lesión inferior del simpático y del parasimpático con lesión superior del pudendo) y en el caso restante no se pudo determinar por la presencia de cambios posquirúrgicos. Los datos neurourológicos de los pacientes con litiasis urinaria se muestran junto con el del resto de los pacientes con MMC en la Tabla 2.

Con respecto a la forma de vaciado vesical, cinco pacientes $(75 \%)$ realizaban autocateterismos limpios intermitentes, dos $(25 \%)$ eran porta- 


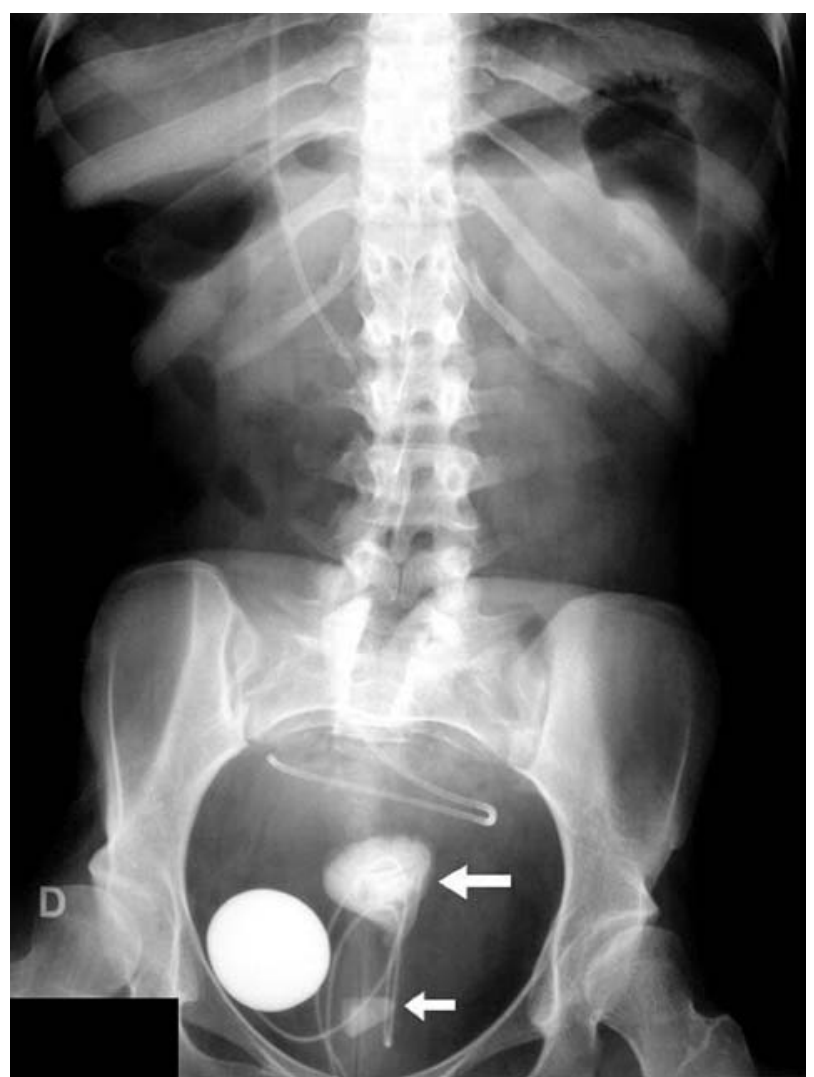

FIGURA 4. Placa simple de abdomen. Litiasis vesical y calcificación del manguito de una prótesis de incontinencia, emigrado al interior de una vejiga ampliada.

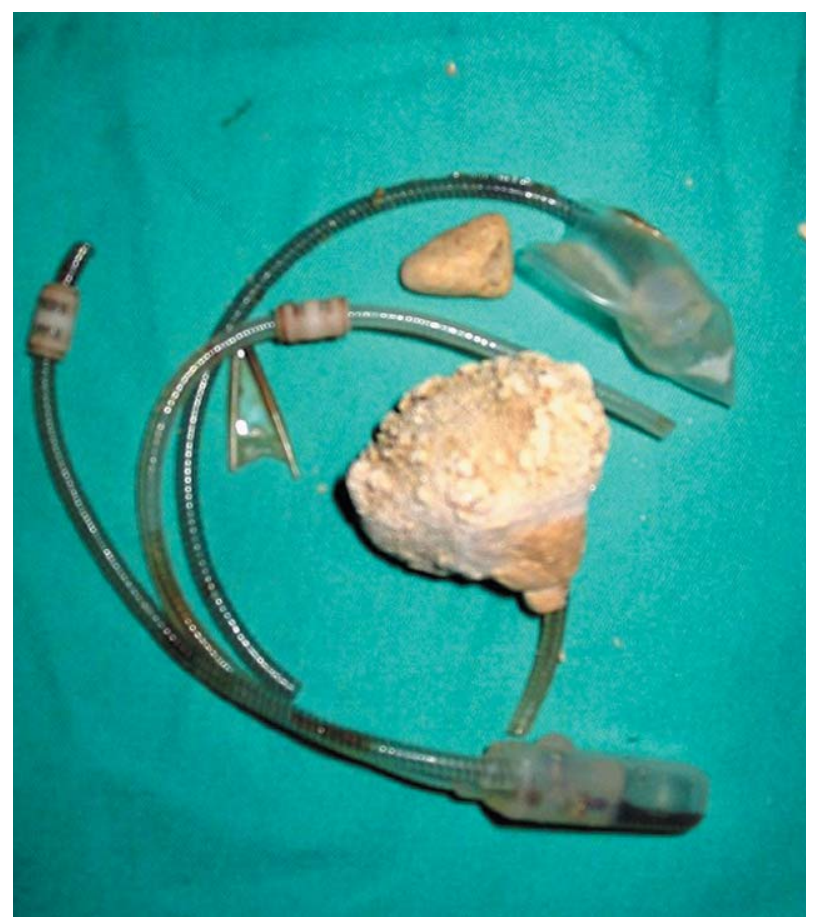

FIGURA 5. Material protésico calcificado extraido, correspondiente al caso de la figura 4.

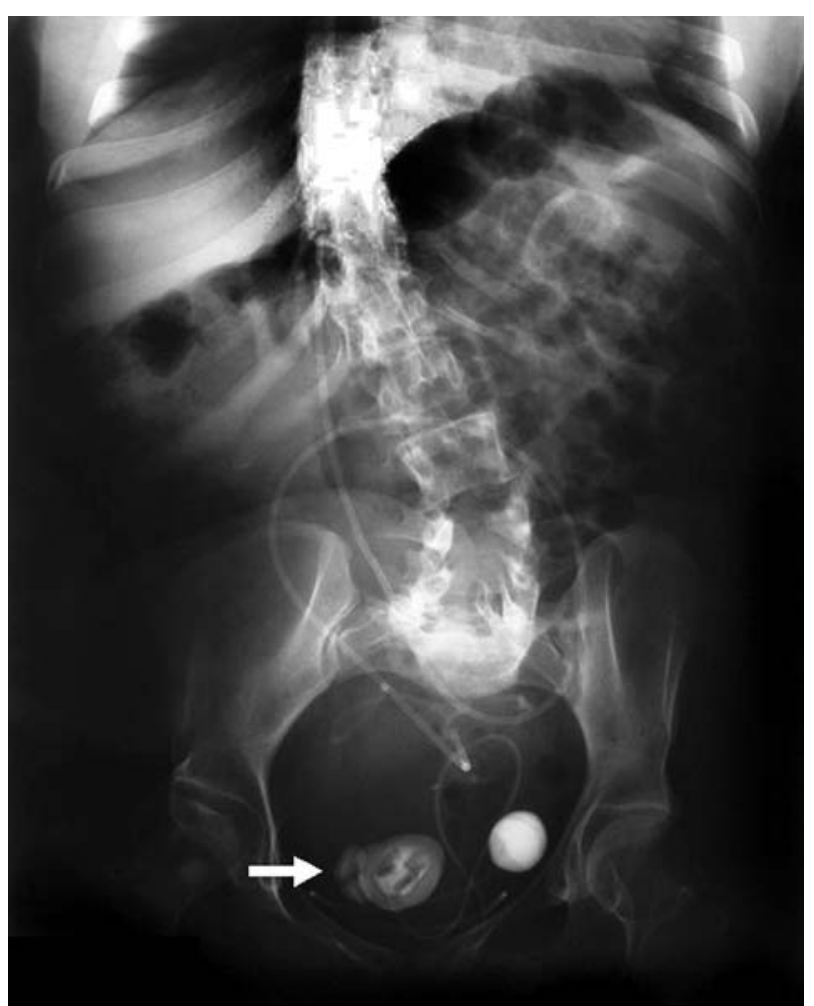

FIGURA 6. Placa simple de abdomen. Calcificación del manguito esfinteriano de una prótesis sin función, emigrado a vejiga. Se aprecian el reservorio y los tubos de conexión, así como la presencia del catéter de derivación ventriculoperitoneal.

dores de colector externo y uno $(12,5 \%)$ vaciaba sin residuo con prensa abdominal. Todos ellos tenían periódicamente urocultivos positivos que trataban dependiendo del germen y de la clínica que causaran. Los gérmenes cultivados más frecuentemente fueron E. coli, Enterococcus faecalis, Proteus y Klebsiella.

La única manifestación clínica de los cálculos vesicales fue la dificultad en la realización de los autocateterismos en dos ocasiones, y una mayor dificultad de vaciado en el paciente que orinaba con prensa.

Con respecto al tratamiento, salvo un pequeño cálculo que pudo ser expulsado mediante micción con prensa, en las restantes 18 ocasiones tuvimos que realizar tratamiento quirúrgico de la litiasis vesical, 4 de ellas por vía abierta, bien por la necesidad de retirar componentes de prótesis de incontinencia asociados a la litiasis o por el volumen del cálculo. En las 14 restantes realizamos litotricia electromecánica vía endoscópica sobre 3 pacientes, que recibieron 2,4 y 8 sesiones 


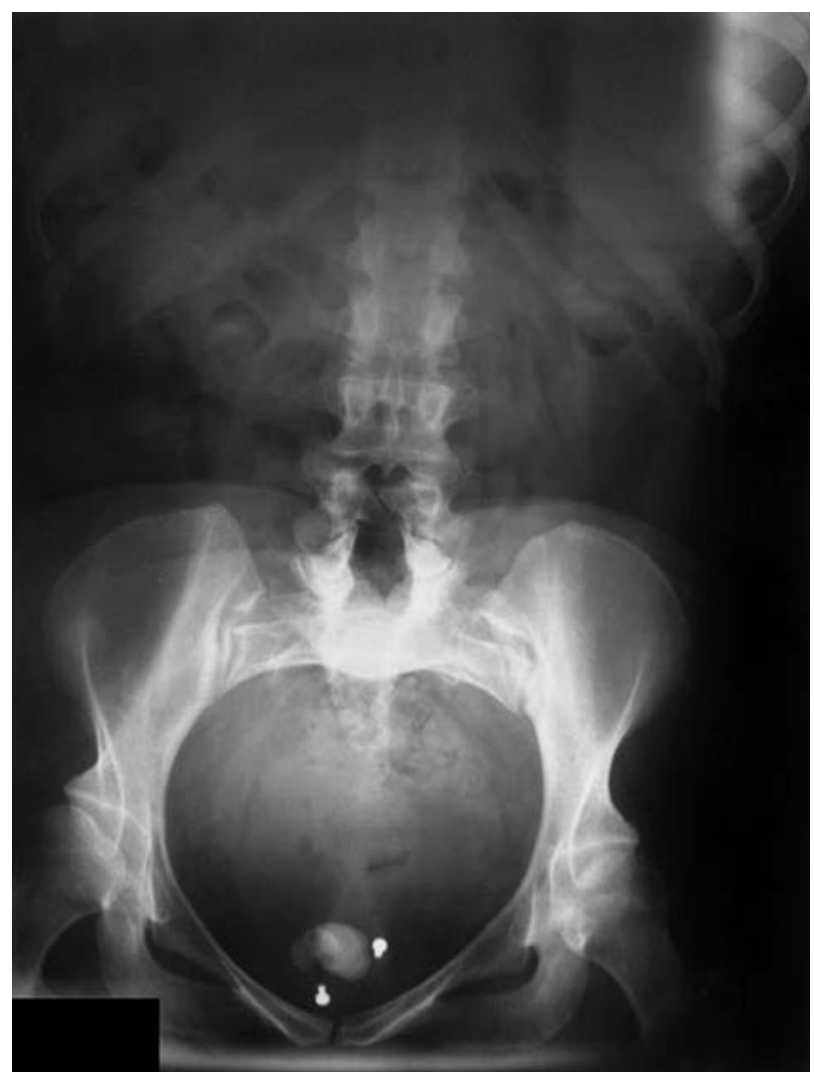

FIGURA 7. Placa simple de abdomen. Litiasis en vejiga ampliada, sobre restos de tubos de conexión de una prótesis terminados en tapones metálicos.

Tabla 2

Características demográficas y neurourológicas

\begin{tabular}{lcc}
\hline & $\begin{array}{c}\text { Pacientes } \\
\text { con MMC } \\
\text { y litiasis } \\
\mathbf{n}(\%)\end{array}$ & $\begin{array}{c}\text { Total de } \\
\text { pacientes } \\
\text { con MMC } \\
\mathbf{n}(\%)\end{array}$ \\
\hline $\begin{array}{l}\text { Número de pacientes } \\
\text { Sexo }\end{array}$ & 10 & 52 \\
$\quad$ - Varón & $6(60)$ & $28(53,8)$ \\
- Hembra & $4(40)$ & $24(46,2)$ \\
Nivel neurológico & & \\
$\quad$ - en o por encima de $\mathrm{L}_{2}$ & $3(30)$ & $11(21,1)$ \\
- entre $\mathrm{L}_{2}$ y S & $5(50)$ & $20(38,4)$ \\
- en o por debajo de $\mathrm{S}_{1}$ & $2(20)$ & $21(40,3)$ \\
Tipo de disfunción neurógena del aparato urinario & & \\
inferior & & $2(3,8)$ \\
- lesión múltiple tipo neurona motora superior & $1(10)$ & $34(65,3)$ \\
- lesión múltiple tipo neurona motora inferior & $7(70)$ & $5(9,6)$ \\
- lesión múltiple mixta & $1(10)$ & $1(1,9)$ \\
- lesión única pura & - & $7(13,4)$ \\
- no valorable & $1(10)$ & $3(5,7)$ \\
\hline
\end{tabular}

respectivamente. En este último caso se asoció en varias ocasiones, resección de un parénquima prostático colonizado por multitud de pequeños cálculos de distintos tamaños en un intento infructuoso de evitar la recidiva litíasica.

El análisis bioquímico de los cálculos vesicales demostró en todo ellos la presencia de fosfato amónico magnésico y de carbonato cálcico en distintas proporciones.

En la Tabla 3 se detallan las características de cada uno de los diez pacientes con MMC y litiasis.

\section{DISCUSIÓN}

Existe un mayor riesgo de litiasis urinaria en los pacientes con defectos del tubo neural que en la población general ${ }^{3,4}$. Los cálculos, aunque pueden formarse a cualquier nivel del aparato urinario, son mas frecuentes en la vejiga, sobre todo si ésta ha sido ampliada ${ }^{3,5}$. Excepcionalmente se han comunicado casos de litiasis vaginales secundarias a estasis urinario ${ }^{6}$ o fístulas vesicovaginales ${ }^{7}$.

La incidencia de la litiasis urinaria en estos pacientes oscila entre el 5\% de los 303 casos revisados por Kondo ${ }^{5}$ y el 10,4\% de los 327 tratados por $\mathrm{Raj}^{8}$. Este mismo autor cifra la litiasis renal en concreto en el 6,1\%, apreciando un aumento del riesgo de la misma con el paso de los años. En nuestra serie tenemos una incidencia de litiasis renal similar $(5,7 \%)$ pero la litiasis en general alcanza el 19,2\%, debido a un aumento en la litiasis vesical. Probablemente esto se deba a que todos nuestros pacientes son adultos, a diferencia de las demás series publicadas que incluyen pacientes de todas las edades. La litiasis urinaria es una de las causas de ingreso hospitalario más frecuentes entre los adultos con MMC ${ }^{9}$.

Se han descrito como factores de riesgo de litiasis renal en estos pacientes, la presencia de bacteriuria, la ectasia ureteropiélica, el reflujo vesicoureteral, la presencia de cicatri- 
Tabla 3

\begin{tabular}{|c|c|c|c|c|c|c|c|}
\hline Caso & $\begin{array}{l}\text { Edad } \\
\text { sexo }\end{array}$ & $\begin{array}{c}\text { Nivel } \\
\text { neurológico }\end{array}$ & $\begin{array}{l}\text { Tipo de disfunción } \\
\text { urinaria inferior }\end{array}$ & $\begin{array}{l}\text { Cirugia sobre el } \\
\text { sistema urinario }\end{array}$ & $\begin{array}{l}\text { Localización } \\
\text { de la litiasis }\end{array}$ & Tratamiento & Evolución \\
\hline 1 & $18 \mathrm{~V}$ & $=\mathrm{L}_{2}$ & $\begin{array}{l}\text { neurona motora } \\
\text { superior }\end{array}$ & - & renal derecha & - & exitus \\
\hline 2 & $40 \mathrm{H}$ & $=\mathrm{L}_{2}$ & $\begin{array}{c}\text { neurona motora } \\
\text { inferior }\end{array}$ & - & renal bilateral & - & $?$ \\
\hline 4 & $20 \mathrm{H}$ & $=\mathrm{S}_{1}$ & $\begin{array}{c}\text { neurona motora } \\
\text { inferior }\end{array}$ & $\begin{array}{c}\text { ampliación vesical } \\
\text { AMS-800 }\end{array}$ & vesical & $2 \mathrm{LE}$ & recidiva \\
\hline 5 & $26 \mathrm{H}$ & $\mathrm{L}_{5}-\mathrm{S}_{1}$ & $\begin{array}{c}\text { neurona motora } \\
\text { inferior }\end{array}$ & AMS-800 & vesical & $\begin{array}{l}\text { cistolitotomía } \\
\text { y retirada AMS }\end{array}$ & 3 años $\sin$ recidiva \\
\hline 7 & $22 \mathrm{~V}$ & $\mathrm{~L}_{5}-\mathrm{S}_{1}$ & $\begin{array}{c}\text { neurona motora } \\
\text { inferior }\end{array}$ & AMS-800 & vesical & $\begin{array}{c}\text { cistolitotomía } \\
\text { y retirada AMS }\end{array}$ & 8 años sin recidiva \\
\hline 8 & $19 \mathrm{H}$ & $\mathrm{L}_{5}-\mathrm{S}_{1}$ & no valorable & $\begin{array}{c}\text { ampliación vesical } \\
\text { AMS-800 }\end{array}$ & vesical & $\begin{array}{l}\text { cistolitotomía } \\
\text { y retirada AMS }\end{array}$ & 2 años $\sin$ recidiva \\
\hline 9 & $25 \mathrm{~V}$ & $\mathrm{~L}_{5}-\mathrm{S}_{1}$ & $\begin{array}{c}\text { neurona motora } \\
\text { inferior }\end{array}$ & - & vesical & cistolitotomia & 2 años $\sin$ recidiva \\
\hline 10 & $23 \mathrm{~V}$ & $\geq \mathrm{S}_{1}$ & múltiple mixta & - & vesical & expulsión & 3 años sin recidivas \\
\hline
\end{tabular}

ces renales y la reconstrucción quirúrgica del aparato urinario inferior, así como un nivel neurológico torácico de la lesión ${ }^{8}$. Nuestros 3 pacientes con litiasis renal tenían un nivel neurológico en o por encima de $\mathrm{L}_{2}$.

Como factores de riesgo de litiasis vesical se ha identificado la infección urinaria, la presencia de sondas a permanencia y la existencia de una ampliación vesical. Kondo demostró que la litiasis vesical era 10 veces más prevalente en los pacientes con MMC portadores de enterocistoplastia $^{5}$. Esta formación de cálculos en la vejiga ampliada, favorecida en los pacientes inmóviles con déficit sensoriales, desciende significativamente si se adoptan protocolos de irrigación ${ }^{10}$. En nuestra serie también fue origen de litiasis vesical, la persistencia de una prótesis de incontinencia afuncionante, o de algunos de sus componentes. Es conocida la tendencia de cualquier cuerpo extraño paravesical a emigrar al interior de la vejiga, por lo que no debe sorprender que lo mismo ocurra con componentes de prótesis de incontinencia sin función. El diagnóstico se ve dificultado por la falta de expresividad clínica y por la difícil visualización con las técnicas de imagen. El déficit sensitivo de estos pacientes, junto con su disfunción vesicouretral, hace que generalmente no refieran molestias por la presencia de sus cálculos aunque estos alcancen grandes tamaños ${ }^{11}$. La aparición de dificultad en el autosondaje debe hacer sospechar la posibilidad de litiasis vesical, como ocurrió en dos de nuestros casos.

Una parte importante de los cálculos en cualquier lesionado medular no es visible en una placa simple de abdomen. Linsenmeyer comprobó que tan solo el $20,9 \%$ de los cálculos vesicales diagnosticados mediante cistoscopia eran visibles en este tipo de placas, concluyendo que esta exploración no es un método fiable en la detección de la litiasis vesical en lesionados medulares $^{12}$. En el caso del paciente con MMC, a su lesión medular se añaden las deformidades óseas pélvicas y su frecuente cifoescoliosis, lo que dificulta aún mas la localización tanto radiológica como ecográfica de sus cálculos y de patologías asociadas a éstos, como ilustra el caso comunicado por Vaidyanathan de extrusión lumbar de un cálculo renal complicado en un adulto con MMC por un error diagnóstico ${ }^{13}$. 
El tratamiento de la litiasis en los pacientes con MMC también presenta ciertas peculiaridades. La litotricia extracorpórea por ondas de choque (LEOC) es tolerada generalmente bien por los lesionados medulares, aunque la expulsión de los fragmentos litiásicos puede ser lenta ${ }^{14}$. En cualquier tipo de tratamiento, debe recordarse la frecuente alergia al látex en el MMC para tomar las medidas oportunas ${ }^{15}$. Cuando a éste se asocia una severa cifoescoliosis se incrementa el riesgo de complicaciones secundarias a daño renal por mala visualización ${ }^{16}$. Nosotros no pudimos tratar mediante LEOC un cálculo renal en un paciente con severa cifoescoliosis, dada la anormal localización anatómica del riñón afecto. Posiblemente también se pueda tratar la litiasis vesical mediante LEOC en los afectos de MMC, al igual que se ha hecho en otros lesionados medulares ${ }^{17}$, completándola con la evacuación endoscópica de los fragmentos litíasicos prescindiendo de la necesidad de anestesia.

Cualquier tratamiento quirúrgico en estos pacientes debe realizarse bajo anestesia general, por la imposibilidad de realizar técnicas de raquianestesia. Sus deformidades óseas a menudo complican la colocación en la mesa del quirófano y dificultan las vías de acceso que pudieran ser requeridas. Estas deformidades pueden incluso imposibilitar la realización de una anestesia general ${ }^{18}$ haciendo imprescindible el diagnóstico temprano de cualquier la litiasis. Se debe considerar que muchos de estos enfermos son portadores de válvulas de derivación ventriculoperitoneales.

La nefrolitotomía percutánea es un método de tratamiento eficaz tanto en pacientes con MMC y derivación urinaria ${ }^{19}$ como en aquellos que presentan cálculos en su vía urinaria nativa ${ }^{20}$. Se recomienda recoger muestras de orina piélica para cultivo durante su realización. En cuanto al tratamiento endoscópico de la litiasis vesical hay que recordar que la alterada dinámica miccional de estos pacientes recomienda la completa extracción de los fragmentos litíasicos.

La recidiva litiásica fue frecuente en nuestra serie, al igual que en otras $\operatorname{publicadas}^{3,8}$, y se debe fundamentalmente a la persistencia de factores de riesgo difícilmente corregibles. El conoci- miento de los mismos obligará a tomar medidas dirigidas a su supresión y contribuirá a un seguimiento más apropiado de los pacientes.

\section{CONCLUSIONES}

La litiasis urinaria es más frecuente entre los adultos nacidos con MMC que entre la población general, y habitualmente se presenta como cálculos vesicales. A los factores de riesgo ya descritos en su desarrollo, habría que añadir la persistencia de componentes de una prótesis de incontinencia sin función. La frecuente persistencia de los factores favorecedores hace de la recidiva algo habitual.

Su diagnóstico se ve dificultado por la lesión neurológica presente y por su difícil localización con las técnicas de imagen, por la especial configuración anatómica que presentan casi todos estos pacientes. Esta configuración anómala junto a la frecuente alergia al látex y la imposibilidad de realizar ciertas técnicas anestésicas, dificultan el tratamiento tanto quirúrgico como por ondas de choque. Por todo esto se hace necesario el seguimiento urológico de los pacientes con objeto de lograr un diagnóstico temprano de su litiasis que facilite su tratamiento y evite complicaciones.

\section{REFERENCIAS}

1. Shurtleff DB, Hayden PW, Chapman WH, Broy AB, Hill ML: Myelodysplasia. Problems of long-term survival and social function. West J Med. 1975;122:199.

2. Verdú Tartajo F: Caracterización neurourológica del mielomeningocele en el paciente adulto. Tesis Doctoral. Facultad de Medicina Universidad Complutense de Madrid. 2005.

3. Gros DAC, Thakkar RN, Lakshmanam Y, Ruffing V, Kinsman SL, Docimo SG: Urolithiasis in spina bifida Eur J Pediatr Surg. 1998;8 Suppl I:68-69.

4. Franco I, Levitt SB: Urolithiasis in the patient with augmentation cystoplasty: pathogenesis and management. AUA Update series 1997; vol 16, lesson 2.

5. Kondo A, Gotoh M, Isobe Y, Kimura K, Kamihira O, Matsuura O : Urolithiasis in those patients with myelodysplasia. Nippon Hinyokika Gakkai Zasshi. 2003Jan;94(1):1519.

6. Petrillo CR, Borrelli M, Botelho C: Primary vaginal calculus in a patient with meningomyelocele. Arch Phys Med Rehabil. 1981 62(5):227-229.

7. Sant GR, Conley GR, Klauber GT. Vaginal calculus in a female with myelodysplasia. Urology. 1983;22(3):294-296.

8. Raj GV, Bennett RT, Preminger GM, King LR, Wiener JS: The incidence of nephrolithiasis in patients with spinal neural tube defects. J urol 1999; 162:1238-1242.

9. Kinsman SL, Doehring MC. The cost of preventable conditions in adults with spina bifida. Eur J Pediatr Surg. 1996; 6 suppl I:17-20. 
10. Hensle TW, Bingham J, Lam J, Shabsigh A. Preventing reservoir calculi after augmentation cystoplasty and continent urinary diversion: the influence of an irrigation protocol. BJU Int. 2004; 93(4):585-587.

11. Gacci M, Cai T, Travaglini F, Rizzo M, Bartoletti R Carini M. Giant stone in enterocystoplasty Urol Int. 2005;75(2): 181-183.

12. Lisenmeyer MA, Lisenmeyer TA: Accuracy of bladder stone detection using abdominal x-ray after spinal cord injury. J Spinal Cord Med. 2004;27(5):438-442.

13. Vaidyanathan S, Hughes PL, Soni BM, Singh G, Mansour P, Sett P: Unpredicted spontaneous extrusion of a renal calculus in a adult male with spina bifida and paraplegia: report of a misdiagnosis. Measures to be taken to reduce urological errors in spinal cord injury patients. BMC Urol. 2001;20(1):3.

14. Niedrach WL, Davis RS, Tonetti FW, Cockett AT : Extracorporeal shock-wave lithotripsy in patients with spinal cord dysfunction. Urology. 1991;38(2):152-156.

15. Martinez Jabaloyas JM, Broseta Rico E, Ruiz Cerdá JL, Sanz Chiniesta S, Osca Garcia JM, Jiménez Cruz JF: Litotricia extracorporea con ondas de choque en pacientes con derivación urinaria. Actas Urol Esp. 1995;19(2);143-147.

16. Vaidyanathan S, Johnson H, Singh G, Hughes P, Soni BM, Parson KF, Sett P: Atrophy of kidney following extra corporeal shock wave lithotripsy of renal calculus in a paraplegic patient with marked spinal curvature. Spinal Cord 2002;40(11):609-614.
17. Kilciler M, Sumer F, Bedir S, Ozgok Y, Erduran D: Extracorporeal shock wave lithotripsy treatment in paraplegic patients with bladder stones. Int J Urol. 2002; 9(11). 632-634.

18. Vaidyanathan S, Soni BM, Wyndaele JJ, Buczynski AZ, Iwatsubo E, Stoehrer M, Madersbacher H, Peschel R, Sinngh G, Watt JW, Hughes PL: Calculus anuria in a spina bifida patient, who had solitary functioning kidney and recurrent renal calculi. Spinal Cord. 2004 42(1):7-13.

19. Krieger JN, Rudd TG, Mayo ME: Infection stines in patients with myelomeningocele and ileal conduit urinary tract diversion. Arch Phys Med Rehabil. 1985;66(8):360364.

20. Rubenstein JN, Gonzalez CM, Blunt LW, Clemens JQ, Nadler RB: Safety and efficacy of percutaneous nephrolithotomy in patients with neurogenic bladder dysfunction. Urology 2004;63(4):636-640.

Dr. F. Verdú Tartajo

E-mail: ferverdu@pulso.com

(Trabajo recibido el 17 de marzo 2006) 